\title{
REESTRUCTURACIÓN INDUSTRIAL: SUBCONTRATACIÓN INTERNACIONAL, CAMBIO TECNOLOGICO Y FLEXIBILIDAD EN LA MAQUILADORA
}

\author{
Por \\ Ma. del Rocio Barajas Escamilla*
}

\begin{abstract}
RESUMEN
Este artículo ${ }^{1}$ se enfoca especialmente sobre los planteamientos teóricos que han dado sustento al fenómeno de la reestructuración industrial en el mundo y en México; a la explicación de los determinantes de la subcontratación y su relación con el cambio tecnológico; y los sistemas de trabajo flexibles. Para ello, se ha utilizado como marco de estudio la industria maquiladora de exportación en México, con la finalidad de observar dicha relación, en la visión de que la evolución y desempeño de esta industria no sólo sea contemplada en forma aislada y como producto de las condiciones internas del país, sino fundamentalmente como resultado de una situación específica de la economía internacional. Como consecuencia de la reestructuración productiva, se está dando una mayor segmentación de los procesos productivos y esto a la vez estimula el crecimiento de las actividades de subcontratación en países en desarrollo como México. Actualmente en la maquiladora mexicana se incorporan nuevas tecnologias, a la vez que se instrumentan los últimos sistemas organizacionales que han venido a revolucionar la manufactura, la cual se caracteriza por la dualidad tecnológica, es decir, introducción de tecnología microelectrónica, que combina ensamble simple y tradicional.
\end{abstract}

\section{ABSTRACT}

The main subject of this article is a discussion of the theory (es) wich give to the support phenomenon of the industrial re-structuring in Mexico and the world; the author attempts to give an explanation of the determinants of subcontracting and their relation to technological change and flexible work systems. The export maquiladora industry in Mexico is studied in order to observe the process and considering that the evolution of this industry is not just a singular product of intemal conditions seem the country, but fundamentally as a result of a specific situation in the international economy.

As a consequence of the industrial re-structuring, a larger segmentation of the productive process occurs, which increases subcontracting activities in development countries like Mexico. Presently Mexican maquilas are incorporating new technologies as well as the latest organizational systems which have revolutionized manufacturing. The result is a technological duality, that is, the introduction of microelectronic technology is connection with simple and traditional assembly.

* Investigadora del Departamento de Estudios Económicos del colep en Tijuana, B. C.

1 Se agradece la asistencia de investigación de Silvano Enrique Gastélum y el apoyotécnico de Raúl Borja. 


\section{REESTRUCTURACIÓN PRODUCTIVA Y SUBCONTRATACIÓN INTERNACIONAL}

En el análisis de la reestructuración industrial en México debe considerarse que su crecimiento y expansión constituye un fenómeno emergente de la competencia internacional entre las empresas por ganar más espacios en los mercados, recurriendo para ello, entre otros, al mecanismo de subcontratación internacional.

El mecanismo de subcontratación se encuentra inmerso a la vez en un proceso mayor, la internacionalización del capital, que constituye la respuesta a la crisis que afecta a países altamente desarrollados como Alemania, Japón y Estados Unidos, quienes participan en una guerra por los mercados y como parte de su estrategia recurren al proteccionismo.

De tal forma que, en el contexto de la internacionalización del capital, la selección de espacios geográficos donde se realiza dicha subcontratación se ve influida por factores de localización que consideran las condiciones optimas para ahorrar costos, incrementar su rentabilidad y facilitar sus estrategias de penetración en los mercados más importantes del mundo.

En México, los procesos de subcontratación juegan un papel de primer orden en el nuevo patrón de acumulación de capital y proyecto de industrialización, en el cual se priorizan las activiades que promueven las exportaciones. En tales condiciones, los espacios regionales que cuentan con condiciones propicias para el intercambio internacional, como es el caso de la región fronteriza norte, adquieren una posición estratégica en los objetivos de política económica nacional.

Otros factores de localización que son importantes tienen que ver, por un lado, con los altos costos que enfrentan las compañías no competitivas ${ }^{2}$ en cuanto a la tierra, los servicios, los estrictos criterios de control ambiental, los altos costos de reciclamiento y de manejo de desechos tóxicos y los elevados salarios en el trabajo calificado; y por otro lado, la búsqueda de los países de la cuenca del Pacífico, y el interés de los europeos para penetrar en el mismo mercado, ${ }^{3}$ para evadir las prácticas proteccionistas a las que está recurriendo Estados Unidos para resolver su problema de deficit comercial. De ahí que resulte sumamente atractivo su traslado a países como México con bajos costos de operación y legislaciones sumamente flexibles.

2 Principalmente empresas de origen norteamericano.

3 Propiciando un porcentaje importante de inversiones de diverso origen, pero principalmente, japonés, coreano, holandés, inglés y franó́s. 


\section{LA REESTRUCTURACIÓN Y RECONVERSIÓN PRODUCTIVA}

Para arribar a una explicación coherente acerca de la naturaleza de los cambios que han ocurrido en México en materia de reconversión productiva (que están llevando a cabo las empresas filiales y subcontratistas de las más importantes multinacionales), ${ }^{4}$ es necesario ubicar el nuevo carácter de la economía internacional y el proceso de reestructuración industrial en el mundo.

Tal y como lo señalan Castells y D'Andrea (1990), este proceso no sólo abarca el comercio, sino también los movimientos de capital, fuerza de trabajo, información, tecnología y la organización del mismo proceso productivo. Por lo tanto, la internacionalización de las economías nacionales es resultado del agotamiento de sus mercados, lo que obliga a los países más desarrollados a participar en forma creciente en este proceso como una de las principales estrategias para mantener su competitividad.

Continuando con el argumento de Castells y D'Andrea, la década de los noventa se caracteriza por la interrelación de tres factores básicos: a) la creciente importancia que están teniendo en el desarrollo de las economías nacionales, su participación en los mercados internacionales; b) la revolución tecnológica en productos y procesos basados en la innovación microelectrónica y c) la reestructuración de las economías que están llevando a cabo los países desarrollados y sus efectos en las políticas de ajuste en los países en desarrollo.

Estos tres factores constituyen en conjunto la base del nuevo paradigma del desarrollo económico que por primera vez está involucrando un verdadero proceso de globalización-internacionalización. Con este proceso de reestructuración se busca recuperar la productividad económica y su crecimiento estable.

Para llevar a cabo dicha reestructuración, los países desarrollados han recurrido a la aplicación de políticas macroeconómicas tales como: el control salarial, la desregulación de los marcos legales para las empresas, la descentralización administrativa, la reducción del gasto social, el aumento en gastos militares, una política monetaria estricta y el énfasis sobre la competitividad internacional. Con algunas diferencias, estas mismas políticas están siendo generalizadas en los países en desarrollo.

La experiencia de los años cincuenta y sesenta demostró, según Druker, que el desarrollo económico ha sido desigual y muy selectivo y que las primeras políticas de desarrollo que se dieron después de la segunda guerra

4 El concepto multinacional en este caso tiene la connotación de la firma matriz que es de donde emanan las estrategias de producción, comercialización y organización que posteriormente se extienden a sus filiales y subcontratistas en otros países. 
mundial - como fueron la exportación de productos de mano de obra industrial barata y la protección de la industria naciente - se fueron abandonando en los países centrales a finales de los años setenta por considerar que la mano de obra barata es improductiva y de muy baja calidad y muy difícilmente puede competir en los mercados internacionales (Druker, 1990).

La reestructuración industrial fue iniciada por países como Japón, que según el mismo autor fue el primer país en comprender que el adiestramiento, un invento norteamericano, elevaba la productividad de los trabajadores sin tener que aumentar salarios y que para salir del subdesarrollo era necesario producir con calidad. De tal forma que variables como calidad y tiempo de entrega igualaron en importancia a la variable "precio".

En los países centrales, esta política de bajos salarios con altos niveles de adiestramiento de la mano de obra no logró sostenerse, debido al reclamo del movimiento obrero organizado para obtener un reconocimiento salarial acorde con los altos niveles de productividad alcanzados. Una vez que dichos países se convirtieron en productores con altos salarios, promovieron en países no desarrollados como México, Tailandia y Malasia la política de bajos salarios.

Como consecuencia de la reestructuración industrial que los países capitalistas desarrollados experimentan, la subcontratación internacional entra a una nueva fase que se caracteriza por el surgimiento de la llamada "dualidad tecnológica", cuyo significado concreto es la coexistencia, dentro de un mismo modelo de acumulación de capital, de procesos productivos que se basan en el uso de tecnología alta y media y los que recurren principalmente a la ocupación de fuerza de trabajo.

\section{REESTRUCTURACIÓN PRODUCTIVA, CAMBIO TECNOLÓ- GICO Y PRODUCTIVIDAD}

En el trabajo de Castells y D'Andrea (1990) se señala que hacia finales de los años ochenta y principios de los noventa, las nuevas fuentes de desarrollo económico se han vinculado de manera más estrecha con las capacidades científicas y administrativas establecidas en las sociedades, instituciones y organizaciones. De tal forma que constituyen los elementos clave en el proceso de reconversión productiva.

Con la aplicación de la microelectrónica a las máquinas-herramienta se posibilita una estrecha coordinación entre las funciones de la empresa (producción, comercialización, administración), logrando reaccionar de manera más eficiente ante los cambios del mercada y posibilitando el incremento de la productividad. 
La reconversión productiva significa la conversión de una economía rígida a una de variedad, a través de dos estrategias fundamentales: la flexibilidad y la integración. Estos conceptos permiten a diversos autores (Mertens, Coriat, Boyer) explicar la naturaleza de los cambios recientes que se suscitan en el mundo, como respuesta a las nuevas necesidades que genera el comercio internacional.

Esta reconversión la explica Coriat (1988) desde la perspectiva del análisis de costos, donde los costos medios que se alcanzan en las firmas que participan de economías de variedad (firmas multiproducto) son inferiores a la suma de los costos medios que se logran en las economías rígidas (empresas monoproductoras).

La transición de una economía rígida a una de variedad resulta, como lo señala Mertens (1988), de la introducción de tecnología programable vinculada a nuevos diseños de productos y del incremento en los niveles de calificación, conocimiento y responsabilidad de los trabajadores, de tal forma que adquieren un carácter polivalente, es decir, se encuentran en posibilidades de desarrollar nuevas tareas, tomar iniciativas y decisiones y se capacitan para trabajar en grupo.

Para Robert Boyer (1990) los primeros indicios de "flexibilización" en la producción masiva se dieron en los años veinte, como un mecanismo de adaptación del fordismo a la renovación acelerada de productos de consumo. El carácter dinámico que adquiere esta renovación requiere que la flexibilidad no se reduzca al plano de la producción, es decir, no basta modificar las prácticas del trabajo y la administración de las relaciones de trabajo, sino también todas las estrategias empresariales.

La flexibilidad se conceptúa, según Piore (1990), desde dos ópticas distintas: la primera, conocida como flexibilidad descendente que parte del supuesto de que las medidas básicas para estimular la productividad de los trabajadores están directamente relacionadas con los costos de producción a través de establecer bajos salarios, aumentar las horas de trabajo y exigir un mayor grado de esfuerzo laboral; la segunda definición está relacionada con la organización de la producción y se lleva a cabo, incluso, en pequeñas firmas con un proceso de producción altamente variable, utilizando una serie de recursos muy calificados capaces de diversificar los productos según la demanda del mercado.

En cuanto a la "integración" como segundo componente de la economía de variedad, concierne a toda la técnica tendiente a convertir los tiempos muertos de producción en tiempos efectivamente productivos, sea que tales tiempos se refieran al trabajo, a la utilización de máquinas y/o al tiempo de almacenamiento de materias primas y de productos en proceso. 
En relación con el incremento en la productividad, Mertens afirma que en el pasado la base para estimular la productividad del trabajo y del capital era, por un lado, la automatización rígida y, por otro, la generación de economías de escala en donde al trabajador le correspondía una función específica e invariable.

Lo anterior dificulta la eliminación de los tiempos muertos del equipo, así como el deslindamiento de responsabilidades en cuanto al control de calidad del proceso y del producto, y genera problemas para establecer flujos de información entre el cuerpo de decisión de la empresa y el frente de trabajo.

Actualmente, como lo afirman Castells y D'Andrea, la manufactura auxiliada por la computación permite la formación de una línea de montaje mundial, mientras que la integración de las operaciones administrativas, a través de los sistemas de telecomunicaciones e información, posibilita la administración unificada de actividades especialmente lejanas.

Esta difusión propicia la "división tecnológica del trabajo" que a su vez permite generar aumentos en la productividad, a pesar de no incrementar los costos de empleo y sin la necesidad de abrir nuevos mercados.

En apoyo a las posiciones anteriores, Coriat afirma que las técnicas taylorianas y fordistas de organización del trabajo han dejado de ser la base para la obtención de ganancias de productividad. En su lugar, el origen de la ganancia en productividad es atribuida al hecho de que varios factores productivos puedan ser utilizados simultáneamente para fabricar diferentes productos.

En relación con los sistemas flexibles de trabajo la fuerza laboral adquiere un nuevo rol, el cual se inscribe en la llamada "innovación blanda", donde la base de su participación en la "gestión de calidad" se logra propiciando la participación de los trabajadores en los ć́rculos de calidad, estadísticas de control de proceso y equipos de trabajo.

En estos sistemas flexibles, la existencia del trabajador polivante es un elemento fundamental; su significado más concreto reside en el hecho de que un solo trabajador logra un nivel de adiestramiento tal que lo capacita para desempeñar diversas tareas y/o actividades dentro de la línea.

Esta polivalencia es estimulada por los propios empresarios como parte de las condiciones necesarias para la flexibilidad y es determinante para la fijación de salarios; es decir, ahora no se reconoce la magnitud del trabajo y esfuerzos realizados por el obrero, sino el nivel de conocimiento y capacitación adquirida para funcionar de acuerdo con las necesidades del mercado.

Después de lo antes expuesto se puede concluir que la conversión de sistemas de trabajo inflexible a sistemas de trabajo flexible ha propiciado la reorganización del proceso productivo y del trabajo, modificando el 
contenido de ambos, de tal forma que la difusión de nuevas tecnologías a las empresas filiales es lenta y selectiva, no así el caso de la difusión de las nuevas prácticas empresariales que rápidamente se han convertido en la base para elevar la productividad.

\section{REESTRUCTURACIÓN INDUSTRIAL Y CARACTERÍSTICAS DEL CRECIMIENTO DE LA INDUSTRIA MAQUILADORA DE EXPORTACIÓN}

El crecimiento sustancial de la maquiladora en la frontera norte se explica en gran medida por la reestructuración industrial que ha tenido como principales centros de relocalización las nuevas zonas de procesamiento de exportaciones (ZPE) de los países menos desarrollados, entre ellos México.

En la industria maquiladora la incorporación de nuevas tecnologías y de organización de los procesos productivos no han desestimulado el crecimiento del empleo en esta industria, de ahí que se mantenga con altas tasas de crecimiento.

Algunos datos que dan cuenta de la evolución de las actividades industriales en la frontera norte son los siguientes: en 1974 la maquiladora de exportación generó 75,947 empleos en el país, en 1990 éstos ascendieron a 460,293, de los cuales el $95 \%$ se ha localizado a lo largo de la frontera norte (véase cuadro 1); entre 1976 y 1981 el empleo creció a un ritmo de $11.9 \%$, incrementándose a $17.7 \%$ durante el periodo 1983-1989.

En 1990, la industria maquiladora contribuyó con el 18\% del empleo industrial del país y con 3,656 millones de dólares por concepto del valor agregado que se suman a la balanza comercial del país.

Es importante señalar que del total de la inversión extranjera en México, en 1989 el $63 \%$ correspondió al sector industrial, del cual el $80 \%$ fue de origen estadounidense y una proporción importante de dicha inversión se aplicó a la industria maquiladora de exportación.

La tendencia que estas empresas exportadoras han seguido en México ha sido la distribución entre un mayor número de ciudades fronterizas y no fronterizas, pero creciendo en términos absolutos en cada ciudad; mientras en 1974 en Ciudad Juárez se concentraba el 24.3\% del empleo maquilador, en 1990 esta proporción había aumentado al $27.47 \%$; en tanto que en Tijuana se pasó de una proporción de 12.2 a 13.78\% en el mismo periodo, y en el caso de Matamoros, en 1974 se concentró el $12.5 \%$ del empleo maquilador, descendiendo en 1990 a $8.3 \%$ (ver cuadro 1).

Las ramas en que actualmente participa la maquiladora tienen que ver con las más dinámicas de la economía internacional; atrás quedaron las décadas en que la rama de mayor crecimiento era la textil y ahora la 
CUADRO 1. Estructura y composición porcentual del empleo por municipios seleccionados en plantas maquiladoras, 1974-1990.

\begin{tabular}{|c|c|c|c|c|c|c|c|c|c|c|c|c|c|c|c|c|c|c|c|}
\hline & 1974 & $\%$ & 1975 & $\%$ & 1976 & $\%$ & 1977 & $\%$ & 1978 & $\%$ & 1979 & $\%$ & & 1980 & $\%$ & 1981 & $\%$ & 1982 & $\%$ \\
\hline Nacional & \multicolumn{2}{|c|}{75947100.00} & \multicolumn{2}{|c|}{67214100.00} & 74496 & 100.00 & 78433 & 100.00 & 90704 & 100.001 & 1113651 & 100.00 & \multicolumn{3}{|c|}{119546100.00} & 130973 & 100.00 & 127048 & 100.00 \\
\hline Mexicali & $7888 \quad 10$ & 10.39 & 6324 & 9.41 & 6604 & 8.86 & 6351 & 8.10 & 6543 & 7.21 & 7965 & 7.15 & \multicolumn{2}{|c|}{7146} & 5.98 & 7628 & 5.82 & 6268 & 4.93 \\
\hline Tijuana & $9276 \quad 12$ & 12.21 & 7844 & 11.67 & 7795 & 10.46 & 7111 & 9.07 & 8778 & 9.68 & 10889 & 9.78 & \multicolumn{2}{|c|}{12343} & 10.32 & 14482 & 11.06 & 14959 & 11.77 \\
\hline Cd. Juárez & $18483 \quad 24$ & 24.34 & 19775 & 29.42 & 23580 & 31.65 & 26792 & 34.16 & 30374 & 33.49 & 36206 & 32.51 & \multicolumn{2}{|c|}{39402} & 32.96 & 43994 & +33.59 & 42695 & 33.61 \\
\hline Nogales & $9827 \quad 12$ & 12.94 & 6794 & 10.11 & 7078 & 9.50 & 7521 & 9.59 & 8849 & 9.76 & 12183 & 10.94 & \multicolumn{2}{|c|}{12921} & 10.81 & 12853 & 9.81 & 12363 & 9.73 \\
\hline Matamoros & $9475 \quad 12$ & 12.48 & 9778 & 14.55 & 10966 & $5 \quad 14.72$ & 11357 & 14.48 & 13443 & 14.82 & 15894 & 14.27 & \multicolumn{2}{|c|}{15231} & 12.74 & 15607 & 11.92 & 14643 & 11.53 \\
\hline No-front. & 50456 & 6.64 & 5302 & 7.89 & 7238 & 9.72 & 7939 & 10.12 & 8574 & 9.45 & 11227 & 10.08 & \multicolumn{2}{|c|}{13338} & 11.16 & 14831 & 11.32 & 14173 & 11.16 \\
\hline \multirow[t]{2}{*}{ Otros front. } & 159532 & 21.01 & 11397 & 16.96 & 11235 & $\quad 15.08$ & 11362 & 14.49 & 14143 & 1559 & 17001 & 15.27 & & 9165 & 16.03 & 21578 & $\quad 16.48$ & 21947 & 17.27 \\
\hline & 1983 & $\%$ & & 1984 & $\%$ & 1985 & $\%$ & 1986 & $\%$ & 1987 & $\%$ & 198 & & $\%$ & & 1989 & $\%$ & 1990 & $\%$ \\
\hline Nacional & 150867 & 100.00 & 199 & 9684 & 100.00 & 211968 & 100.00 & 249833 & 100.00 & 305253 & 3100.00 & D 3694 & & 100.00 & & 429725 & 100.00 & 460293 & 100.00 \\
\hline Mexicali & 7392 & 4.90 & & 0264 & 5.14 & 10876 & 5.13 & 12727 & 5.09 & 16312 & 5.34 & 195 & & 5.29 & & 21374 & 4.97 & 21724 & 4.72 \\
\hline Tijuana & 17423 & 11.55 & & 3047 & 11.54 & 25913 & 12.22 & 30248 & 12.11 & 38575 & 12.64 & 497 & & 13.47 & & 58029 & 13.50 & 63416 & 13.78 \\
\hline Cd. Juárez & 54073 & 35.84 & & 2495 & 36.30 & 77592 & 36.61 & 86526 & 34.63 & 97800 & 32.04 & 41109 & & 30.0 & & 124386 & 28.95 & 126452 & 27.47 \\
\hline Nogales & 13278 & 8.80 & & 5964 & 7.99 & 14539 & 6.86 & 15252 & 6.10 & 19427 & 6.36 & 228 & & 6.19 & & 22635 & 5.27 & 19795 & 4.30 \\
\hline Matamoros & 15639 & 10.37 & & 9454 & 9.74 & 20686 & 9.76 & 23442 & 9.38 & 26994 & 8.84 & 324 & & 8.78 & & 38132 & 8.87 & 38410 & 8.34 \\
\hline No-front. & 16781 & 11.12 & & 3906 & 11.97 & 27304 & 12.88 & 39198 & 15.69 & 8802 & 2.88 & 119 & & 3.2 & & 16676 & 3.88 & 20573 & 4.47 \\
\hline Otros front. & 2628 & 117.42 & & 4554 & 17.30 & 35058 & 16.54 & 42440 & 16.99 & 97343 & 31.89 & $1218 t$ & & 32.98 & & 148493 & 34.56 & 169923 & 36.92 \\
\hline
\end{tabular}

FUENTE: Estadística de la Industria Maquiladora de Exportación, varios años, INEGI. 
encabezan la eléctrica y electrónica y la automotriz; precisamente aquellas en que se ha producido el mayor número de cambios en cuanto a incorporación de nuevas tecnologías y organización de los procesos de trabajo. Para 1990 el $48.4 \%$ se distribuye entre la electrónica, aparatos eléctricos y equipo de transporte y el resto en ramas como prendas de vestir, productos de madera, de metal, etcétera (ver cuadro 2).

En la maquiladora mexicana, el empleo de mujeres ha disminuido más rápidamente que en otras $\mathrm{ZPE}$, aunque sigue constituyendo cerca de la mitad de la fuerza de trabajo en el sector y también predominan en aquellas empresas en que se incorpora el cambio tecnológico y los sistemas de organización flexible en el trabajo. Se encuentra que en 1985, las mujeres representaban el $54 \%$ de los obreros de la maquiladora de Ciudad Juárez, el $56.2 \%$ en Tijuana y el $63 \%$ en Matamoros; esta proporción disminuye para 1990 al 43,45 y $58 \%$ respectivamente (ver cuadro 3 ).

Esta nueva tendencia en la composición por sexo está relacionada con el surgimiento de nuevas actividades de maquila que requieren de la ocupación de varones, así como con la rotación en el empleo (que en lugares como Tijuana y Ciudad Juárez llega a ser superior al $10 \%$ mensual) que caracteriza la ocupación de mujeres jóvenes, cuya permanencia en el trabajo está en gran medida en función de su ciclo de matrimonio y reproducción. El desequilibrio en el empleo que este proceso ha generado en las empresas puede haberlas llevado a ocupar los puestos vacantes con trabajadores de cualquier sexo; sin embargo, cuando se analiza esta industria por ramas, la participación de las mujeres sigue siendo mayoritaria en casos como la clectrónica y textil.

Es importante resaltar que, a pesar de que en México el trabajo femenino no tiene las proporciones que en otros países, la ocupación de mujeres sigue siendo un rasgo característico de la industria maquiladora y es de esperarse que en el contexto de una mayor liberalización y expansión de algunas ramas del sector manufacturero, su nivel de ocupación se mantenga.

\section{REESTRUCTURACIÓN Y DIVERSIDAD TECNOLÓGICA EN LA INDUSTRIA MAQUILADORA EN TIJUANA}

Bajo el entorno de la reestructuración productiva, el rápido crecimiento de la industria maquiladora llevó a creer que la maquiladora podía ser portadora de tecnologf́a de punta, venta de insumos nacionales, capacitación de la fuerza de trabajo, fortalecimiento del sector industrial, desarrollo regional, etcétera.

Como ya se señaló, en décadas anteriores la totalidad de las plantas maquiladoras enfatizaban exclusivamente el uso intensivo de mano de 
CUADRO 2. Estructura y composición porcentual del empleo en plantas maquiladoras por rama de actividad, 1979-1990.

\begin{tabular}{lrrrrrrrrrrrr}
\hline & 1979 & $\%$ & 1980 & $\%$ & 1981 & $\%$ & 1982 & $\%$ & 1983 & $\%$ & 1984 & $\%$ \\
\hline Total nacional & 111365 & 100.0 & 119546 & 100.0 & 130973 & 100.0 & 127048 & 100.0 & 150867 & 100.0 & 199684 & 100.0 \\
Alimentos & 1481 & 1.3 & 1393 & 1.2 & 1572 & 1.2 & 1618 & 1.3 & 1898 & 1.3 & 1753 & 0.9 \\
Textil & 14892 & 13.4 & 14256 & 11.9 & 14278 & 10.9 & 11891 & 9.4 & 12885 & 8.5 & 15161 & 7.6 \\
Calzado y cuero & 1546 & 1.4 & 1531 & 1.3 & 1821 & 1.4 & 2043 & 1.6 & 2779 & 1.8 & 3648 & 1.8 \\
madera y metal & 3442 & 3.1 & 3163 & 2.6 & 3236 & 2.5 & 3032 & 2.4 & 4752 & 3.1 & 6201 & 3.1 \\
Equipo de transporte & 5035 & 4.5 & 7100 & 5.9 & 10108 & 7.7 & 11537 & 9.1 & 18814 & 12.5 & 28040 & 14.0 \\
Equipo no eléctrico & 1834 & 1.6 & 1834 & 1.5 & 1402 & 1.1 & 1327 & 1.0 & 1514 & 1.0 & 2154 & 1.1 \\
Aparatos eléctricos & 27598 & 24.8 & 28580 & 23.9 & 31801 & 24.3 & 30787 & 24.2 & 32255 & 21.4 & 41691 & 20.9 \\
Electrónica & 30713 & 27.6 & 33530 & 28.0 & 36935 & 28.2 & 35641 & 28.1 & 40002 & 26.5 & 53316 & 26.7 \\
Juguetes & 2454 & 2.2 & 2803 & 2.3 & 2666 & 2.0 & 2565 & 2.0 & 3477 & 2.3 & 6172 & 3.1 \\
Otras industrias & 6843 & 6.1 & 7483 & 6.3 & 7451 & 5.7 & 6948 & 5.5 & 7451 & 4.9 & 9655 & 4.8 \\
Servicios & 4699 & 4.2 & 4820 & 4.0 & 5105 & 3.9 & 5838 & 4.6 & 8088 & 5.4 & 9118 & 4.6
\end{tabular}




\begin{tabular}{|c|c|c|c|c|c|c|c|c|c|c|c|c|}
\hline & 1985 & $\%$ & 1986 & $\%$ & 1987 & $\%$ & 1988 & $\%$ & 1989 & $\%$ & 1990 & $\%$ \\
\hline Total nacional & 211968 & 100.0 & 249833 & 100.0 & 305253 & 100.0 & 369489 & 100.0 & 429725 & 100.0 & 460293 & 100.0 \\
\hline Alimentos & 1855 & 0.9 & 2185 & 0.9 & 2464 & 0.8 & 3442 & 0.9 & 4361 & 1.0 & 3935 & 0.9 \\
\hline Textill & 15089 & 7.1 & 16883 & 6.8 & 19399 & 6.4 & 20289 & 5.5 & 20672 & 4.8 & 20575 & 4.5 \\
\hline Calzado y cuero & 4328 & 2.0 & 4339 & 1.7 & 4942 & 1.6 & 5765 & 1.6 & 7298 & 1.7 & 6165 & 1.3 \\
\hline Madera y metal & 6522 & 3.1 & 9632 & 3.9 & 14349 & 4.7 & 17770 & 4.8 & 21384 & 5.0 & 24413 & 5.3 \\
\hline Equipo de transporte & 36978 & 17.4 & 43055 & 17.2 & 51328 & 16.8 & 64213 & 17.4 & 75765 & 17.6 & 77353 & 16.8 \\
\hline Equipo no eléctrico & 2386 & 1.1 & 3253 & 1.3 & 3787 & 1.2 & 4878 & 1.3 & 5696 & 1.3 & 4960 & 1.1 \\
\hline Aparatos eléctricos & 38994 & 18.4 & 42617 & 17.1 & 43093 & 14.1 & 47789 & 12.9 & 53463 & 12.4 & 46855 & 10.2 \\
\hline Electronica & 48943 & 23.1 & 53878 & 21.6 & 66669 & 21.8 & 79666 & 21.6 & 87079 & 20.3 & 98361 & 21.4 \\
\hline Juguetes & 7265 & 3.4 & 7110 & 2.8 & 9470 & 3.1 & 11443 & 3.1 & 12154 & 2.8 & 10698 & 2.3 \\
\hline Otras industrias & 12473 & 5.9 & 16291 & 6.5 & 21637 & 7.1 & 28771 & 7.8 & 34495 & 8.0 & 35602 & 7.7 \\
\hline Servicios & 11167 & 5.3 & 13048 & 5.2 & 13718 & 4.5 & 13672 & 3.7 & 15269 & 3.6 & 15314 & 3.3 \\
\hline
\end{tabular}

FUENTE: Estadística de la Industria Maquiladora de Exportación, varios años, INEGI. 
CUADRO 3. Composición porcentual de la fuerza de trabajo de la industria maquiladora por municipio 1985 y 1990.

\begin{tabular}{|c|c|c|c|c|c|c|c|c|c|c|}
\hline \multirow{3}{*}{$\begin{array}{l}\text { Año } \\
\text { Localidad }\end{array}$} & \multicolumn{5}{|c|}{1985} & \multicolumn{5}{|c|}{1990} \\
\hline & \multirow{2}{*}{ Total } & \multicolumn{2}{|c|}{ Obreros } & \multirow{2}{*}{$\begin{array}{l}\text { Técnicos de } \\
\text { producción }\end{array}$} & \multirow{2}{*}{$\begin{array}{l}\text { Empleados } \\
\text { admvos. }\end{array}$} & \multirow[t]{2}{*}{ Total } & \multicolumn{2}{|c|}{ Obreros } & \multirow{2}{*}{$\begin{array}{l}\text { Tácnicos de } \\
\text { producción }\end{array}$} & \multirow{2}{*}{$\begin{array}{l}\text { Empleados } \\
\text { admvos. }\end{array}$} \\
\hline & & Homb. & Muj. & & & & Homb. & Muj. & & \\
\hline Nacional & 100.0 & 25.4 & 56.6 & 11.8 & 6.2 & 100.0 & 31.6 & 49.1 & 11.9 & 7.4 \\
\hline Ensenada & 100.0 & 28.8 & 58.2 & 5.3 & 7.7 & 100.0 & 24.4 & 63.4 & 5.9 & 6.3 \\
\hline Mexicali & 100.0 & 26.1 & 56.6 & 11.6 & 5.7 & 100.0 & 29.5 & 49.2 & 13.9 & 7.4 \\
\hline Tecate & 100.0 & 25.6 & 58.8 & 9.9 & 5.7 & 100.0 & 35.5 & 46.1 & 12.3 & 6.1 \\
\hline Tijuana & 100.0 & 27.1 & 56.2 & 10.4 & 6.3 & 100.0 & 36.7 & 45.7 & 10.7 & 6.8 \\
\hline $\mathrm{LaPaz}$ & 100.0 & 8.0 & 72.0 & 10.0 & 10.0 & 100.0 & 15.1 & 72.5 & 7.1 & 5.4 \\
\hline Ciudad Acuña & 100.0 & 32.9 & 55.4 & 7.8 & 3.9 & 100.0 & 38.6 & 45.2 & 11.5 & 4.7 \\
\hline Piedras Negras & 100.0 & 18.8 & 67.8 & 9.9 & 3.5 & 100.0 & 38.9 & 44.3 & 12.2 & 4.5 \\
\hline Ciudad Juárez & 100.0 & 26.3 & 54.1 & 12.8 & 6.8 & 100.0 & 35.5 & 43.1 & 13.3 & 8.1 \\
\hline Agua Pricta & 100.0 & 32.5 & 51.0 & 13.0 & 3.6 & 100.0 & 33.8 & 42.4 & 19.4 & 4.5 \\
\hline Nogales & 100.0 & 37.4 & 44.0 & 13.5 & 5.2 & 100.0 & 41.2 & 38.3 & 14.2 & 6.2 \\
\hline Matamoros & 100.0 & 22.5 & 62.6 & 10.6 & 4.3 & 100.0 & 25.7 & 57.9 & 10.4 & 5.9 \\
\hline Nuevo Laredo & 100.0 & 25.3 & 60.7 & 11.2 & 2.9 & 100.0 & 35.1 & 45.0 & 14.0 & 5.9 \\
\hline Reynosa & 100.0 & 21.3 & 57.4 & 14.9 & 6.4 & 100.0 & 28.8 & 47.1 & 11.4 & 12.7 \\
\hline No fronterizos & 100.0 & 16.7 & 64.9 & 10.3 & 8.1 & 100.0 & 23.7 & 58.7 & 10.3 & 7.4 \\
\hline
\end{tabular}

FUENTE: Estadistica de la Industria Maquiladora de Exportación, varios años, INEGL. 
obra y del ensamble simple; en la década actual, aun cuando esta característica persiste, se combina con el uso de tecnologías medias y con el desarrollo de procesos integrales de manufactura. Un ejemplo de lo anterior son las empresas japonesas y asiáticas en el área de domésticos y televisores y de automóviles en el caso de coinversión norteamericana japonesa.

La diferenciación tecnológica es una peculiaridad propia de una buena parte de las plantas maquiladoras que se han instalado en México en los años más recientes. En 1988, mediante una muestra de 37 empresas maquiladoras de la rama electrónica en Tijuana (Barajas, 1989), se captó que solamente en 13 de esas plantas se utilizaba algún tipo de maquinaria y equipo electrónico (ver cuadro 4), existiendo una amplia variedad entre plantas.

Asimismo se constató que la utilización de tecnología microelectrónica se restringía a ciertas áreas y actividades, que no sustituía el trabajo directo ni se remplazaban hombres por mujeres para la operación de dicha tecnología, continuando el predominio en estas maquiladoras de las largas líneas de ensamble simple, ahora bajo una organización flexible del trabajo, que apunta necesariamente hacia el objetivo de incrementar la productividad.

Con la misma base de datos que se señaló y después de una minuciosa revisión acerca del tipo de maquinaria y equipo incorporado por estas plantas maquiladoras, se encontró que la mayoría se clasifica como "equipo de apoyo para la producción", aun cuando se trate de equipo microelectrónico.

El mayor grado de tecnificación se encontró en las pruebas por computadora para verificar calidad; en la inserción automática en placas de circuitos; en la inserción automática de cuatro tipos, siendo lo más sofisticado el "sistema computadora" que opera la producción de programas de secuencia y de inserción en forma de cintas perforadas que hacen funcionar cada una de las máquinas y la máquina panasert, la cual desarrolla funciones conjuntas de insertadora y secuenciadora de componentes que son tomados por la máquina directamente.

Fuera de los casos señalados, la maquinaria y equipo de mayor uso son las máquinas sencillas de inserción, desgrasadoras, cortadoras, soldadoras, empaquetadoras, de embobinado automático, de lijado, máquina etiquetadora, cepilladora, de enrollado automático, cabezas magnéticas para impresoras, inspección con equipo electrónico, transportadora de equipo eléctrico, transportadora de equipo electrónico, máquina de moldeo (ver cuadro 4).

Una característica general que se encuentra en el uso de maquinaria y equipo microelectrónico es que involucra la realización, por parte del trabajador, de otras actividades simultáneas y paralelas al funcionamiento del equipo; como son por ejemplo realizar las lecturas adecuadas, $\sin$ las cuales no se cumpliría el objetivo de lograr la calidad total del proceso. 
CUADRO 4. Diversidad tecnológica de los procesos productivos por plantas seleccionadas.

\begin{tabular}{|c|c|c|c|c|c|c|c|c|c|c|c|c|}
\hline Equipo & $\begin{array}{l}\text { ón.de } \\
\text { wilad. }\end{array}$ & $\begin{array}{l}\text { Boum's } \\
\text { de Mex. }\end{array}$ & $\begin{array}{l}\text { Commair } \\
\text { Rotron }\end{array}$ & $\begin{array}{l}\text { Componcantes } \\
\text { Ténic, de B.C }\end{array}$ & $\begin{array}{l}\text { Electron. Ensambl. y } \\
\text { Intermal. Circ. Impres. }\end{array}$ & $\begin{array}{l}\text { Ensamblad. } \\
\text { d/Golfo }\end{array}$ & $\begin{array}{c}\text { Ensamb. } \\
\text { d/Precison Calif. }\end{array}$ & $\operatorname{mesc}$ & $\begin{array}{l}\text { Matsushita } \\
\text { Indust. }\end{array}$ & Plamex & Siemein & $\begin{array}{l}\text { Switch Crat } \\
\text { do Mexioo }\end{array}$ \\
\hline Prensas preformadas & $\mathbf{x}$ & & & & & & & & & & & \\
\hline Cortad. de excodentes & $\mathbf{x}$ & & & & & & & & & & & \\
\hline Máquina p/inserción & $\mathbf{x}$ & $\mathbf{x}$ & & & & & & & & & $\mathbf{x}$ & \\
\hline Miq, electron p/soldar & $\mathbf{x}$ & & & & & & $\mathbf{x}$ & & & $\mathbf{x}$ & $\mathbf{x}$ & $\mathbf{x}$ \\
\hline Maquina de presión & $\mathbf{x}$ & & & & & & & & & & & \\
\hline $\begin{array}{l}\text { Prensa p/bobinado } \\
\text { de tomillos }\end{array}$ & $\mathrm{x}$ & & & & & & & & & & & \\
\hline \multicolumn{13}{|l|}{ Banda transportadora } \\
\hline Mliquina desgrasadora & $\mathbf{x}$ & & $\mathbf{x}$ & & & & & & $\mathbf{x}$ & & & \\
\hline Miq. prohadora de fiucgo & $\mathbf{x}$ & & . & & & & & & & & & \\
\hline Máq. contadora autom. & $\mathbf{x}$ & & $\mathbf{x}$ & & & & $\mathbf{x}$ & & & & & \\
\hline Prensa electromagnética & $\mathrm{x}$ & & & & & & & & & & & \\
\hline Míq, enpaquetadora & $\mathbf{x}$ & & & & & & & & & & & \\
\hline Miq. dentetidora de hule & $\mathbf{x}$ & & & & & & & & & & & \\
\hline Embobinadon & $x$ & & $\mathbf{x}$ & & & & & & $\mathbf{x}$ & & $\mathbf{x}$ & \\
\hline Secuenciadora & & & & & & & & & $\mathrm{x}$ & & & \\
\hline Oseiloscopios & & & & & & & & $\mathbf{x}$ & & & & \\
\hline Maq. probad. impressión & $\mathbf{x}$ & & & & & & & & & & & \\
\hline Inserción automítica & & & & & & & & & $\mathbf{x}$ & & & \\
\hline Míq. phquitar rehabe & $\mathbf{x}$ & & & & & & & & & & & \\
\hline Prueba ploomputadora & $\mathbf{x}$ & & & & & & & & & & & \\
\hline Inspec, monitor $\mathrm{p} / \mathrm{t} \mathbf{v}$. & $\mathrm{x}$ & & & & & & & & & & & \\
\hline Míq. de moldeo & & $\mathbf{x}$ & & & & & $\mathbf{x}$ & & & & , & \\
\hline
\end{tabular}




\begin{tabular}{|c|c|c|c|c|c|c|c|c|c|c|c|}
\hline $\begin{array}{l}\text { Admón. de } \\
\text { Maquilad. }\end{array}$ & $\begin{array}{l}\text { Boum's } \\
\text { de Méx. }\end{array}$ & $\begin{array}{l}\text { Comair } \\
\text { Rotron }\end{array}$ & $\begin{array}{l}\text { Componentes } \\
\text { Técnic. de B.C }\end{array}$ & $\begin{array}{l}\text { Electron. } \\
\text { Intermal. }\end{array}$ & $\begin{array}{l}\text { Ensambl. y } \\
\text { Circ. Impres. }\end{array}$ & $\begin{array}{c}\text { Ensamblad. } \\
\text { d/Golfo }\end{array}$ & d/Precis.DA Calif. & $\begin{array}{l}\text { Matsushita } \\
\text { Indust. }\end{array}$ & Plamex & Sierracin & $\begin{array}{l}\text { Switch Craft } \\
\text { de México }\end{array}$ \\
\hline Máq. lijadora & & $\mathbf{x}$ & & & & & & & & & \\
\hline Máq. etiquetadora & & $\mathbf{x}$ & & & & & & & & & \\
\hline Horno de barnizado & & $\mathbf{x}$ & & & & & & & & & \\
\hline \multicolumn{12}{|l|}{ Automático } \\
\hline Ensamblado automático & & $\mathbf{x}$ & & & & & & & & & \\
\hline Remachadora & & $\mathbf{x}$ & & & & & & & & & \\
\hline Máq. cepilladora autom. & & & $\mathbf{x}$ & & & & & & & & \\
\hline Empaque automático & & & & $\mathbf{x}$ & & & & & & & \\
\hline Conteo automático & & & & & $\mathbf{x}$ & & & & & & \\
\hline Sellado automático & & & & & & $\mathbf{x}$ & & $\mathrm{x}$ & & & \\
\hline Máq. aglomeradora & & & & & & $\mathbf{x}$ & & & & & \\
\hline Máq. super mezcladora & & & & & & $\mathbf{x}$ & & & & & \\
\hline Máq. de extrusión & & & & & & $\mathbf{X}$ & & & & & \\
\hline Máq. perforadora & & & & & & $\mathbf{x}$ & & & & & \\
\hline Máq. autom. p/derretir & & & & & & $\mathbf{x}$ & & & & & \\
\hline Máq. de enrrollado & & & & & & & $\mathbf{x}$ & & & & \\
\hline electrónico & & & & & & & & & & & \\
\hline Desarmador neumático & & & & & & & $\mathbf{x}$ & & & & \\
\hline $\begin{array}{l}\text { Maq. alimentadora } \\
\text { de alambre }\end{array}$ & & & & & & & $\mathbf{x}$ & & & & \\
\hline Maq. de preformación & & & & & & & & $\mathbf{x}$ & & & \\
\hline Soldado automático & & & & & & & & $\mathbf{x}$ & & & \\
\hline Recubridora & & & & & & & & $\mathbf{x}$ & & & \\
\hline Limpiadoras & & & & & & & & & & $\mathbf{x}$ & \\
\hline
\end{tabular}

FUENTE: Elaborado por el autor en base a información que las empresas reportan a la SECOFI-Cuestionario L.M. DEE-COLEF, 1988. 
De tal forma, las evidencias encontradas confirman el hecho de que la nueva tecnología funciona como equipo de apoyo al trabajo manual. Es aquí donde sobresale el papel determinante que juega la mujer obrera en los sistemas flexibles de producción, donde la adopción de equipo microelectrónico se sustenta en el aprovechamiento de las cualidades del trabajo desempeñado por la mujer, como una estrategia en esas plantas maquiladoras para elevar sustancialmente la productividad del trabajo.

A pesar de que la subcontratación en México a través de la industria maquiladora de exportación, ha sufrido importantes modificaciones que incluyen la instalación de procesos con mayor grado de sofisticación tecnológica, siguen siendo predominantes las actividades altamente intensivas en ocupación de mano de obra, en donde el desarrollo de la habilidad manual y agudeza de los sentidos del individuo son parte de las características escenciales que conforman el perfil del personal directo de producción en la maquiladora.

La articulación del mercado de trabajo mundial con el mercado de trabajo en la maquiladora se realiza a través de la caída de los salarios reales, particularmente de los no especializados, que corresponden al $85 \%$ de la fuerza de trabajo en esta industria (Barajas, 1989:9).

En una comparación salarial entre diversos países por el periodo de 1975 a $1988,{ }^{5}$ se encuentra que mientras países como Estados Unidos, Canadá y Japón (incluso Corea) mantienen un crecimiento sostenido de sus salarios; países subcontratistas-maquiladores como Brasil, Singapur, Taiwán, Srilanka y México, muestran inestabilidad y caídas salariales importantes.

$\mathrm{Al}$ analizar con detenimiento el caso de México, se observa que en 1975 el costo laboral promedio por hora en la producción manufacturera era de 2 dólares, que es el mismo valor del trabajo en 1988, es decir 13 años después. En el país, la manipulación de los términos de intercambio internacional con base en la política de modificación de tipos de cambio es en gran medida la causa de la inestabilidad salarial que en 1986 conoció su nivel más bajo de las décadas más recientes, 1.50 dólares por hora y en 1981 su nivel más alto, 3.71 dólares por hora (ver cuadro 5).

El desarrollo técnico-científico alcanzado en los años más recientes ha permitido la concresión de nuevas estrategias del capital para mantener un mayor control sobre los procesos de trabajo. Bajo la visión keynesiana el aumento en la productividad responde a la ley de los mercados: "es posible mejores salarios y disminuir el número de horas de trabajo sin dejar de mejorar las condiciones de trabajo y el confort de la casa" (CORIAT, 1982).

5 El cuadro de referencia aparece en la revista Momento Económico no. 49, de enero-febrero de 1990, unAM, México, D.F. 
CUADRO 5. Costo laboral por hora en la producción manufacturera (dólares).

\begin{tabular}{lcccccccccccccc}
\hline País & 1975 & 1976 & 1977 & 1978 & 1979 & 1780 & 1981 & 1982 & 1983 & 1984 & 1985 & 1986 & 1987 & 1988 \\
\hline Estados Unidos & 6.36 & 6.92 & 7.59 & 8.27 & 9.02 & 9.84 & 10.84 & 11.64 & 12.10 & 12.51 & 12.96 & 13.21 & 13.46 & 13.90 \\
Canadá & 5.78 & 6.91 & 7.19 & 7.25 & 7.61 & 8.37 & 9.22 & 10.09 & 10.85 & 10.99 & 10.81 & 11.01 & 11.98 & 13.58 \\
Japón & 3.05 & 3.30 & 4.03 & 5.54 & 5.49 & 5.61 & 6.18 & 5.70 & 6.12 & 6.34 & 6.47 & 9.47 & 11.14 & 13.14 \\
Taiwan & 0.39 & 0.45 & 0.52 & 0.61 & 0.78 & 0.98 & 1.18 & 1.22 & 1.27 & 1.48 & 1.46 & 1.67 & 2.18 & 2.71 \\
Singapur & 0.84 & 0.86 & 0.91 & 1.05 & 1.26 & 1.49 & 1.79 & 1.96 & 2.21 & 2.46 & 2.47 & 2.23 & 2.31 & 2.67 \\
Corea & 0.04 & 0.44 & 0.58 & 0.80 & 1.08 & 1.02 & 1.08 & 1.16 & 1.23 & 1.32 & 1.36 & 1.46 & 1.79 & 2.46 \\
Hong Kong & 0.76 & 0.87 & 1.03 & 1.18 & 1.31 & 1.51 & 1.56 & 1.67 & 1.52 & 1.60 & 1.75 & 1.89 & 2.12 & 2.43 \\
México & 2.00 & 2.14 & 1.77 & 2.11 & 2.41 & 2.96 & 3.71 & 2.55 & 1.85 & 2.06 & 2.09 & 1.50 & 1.57 & 2.00 \\
Brasil & 0.86 & 1.03 & 1.13 & 1.33 & 1.46 & 1.38 & 1.64 & 1.86 & 1.26 & 1.16 & 1.22 & 1.61 & 1.48 & n.d. \\
Israel & 2.25 & 2.38 & 2.69 & 2.57 & 3.31 & 3.79 & 4.17 & 4.45 & 4.87 & 4.65 & 4.06 & 5.20 & 6.34 & n.d. \\
Srilanka & 0.28 & 0.24 & 0.31 & 0.26 & 0.23 & 0.22 & 0.21 & 0.24 & 0.25 & 0.25 & 0.28 & 0.29 & 0.30 & n.d. \\
& & & & & & & & & & & & & & \\
\hline
\end{tabular}

FUENTE: U.S. Department of Labor, Bureau of Labor Statistics, International Comparisons of Hourly Compensation Costs for Production Workers in Manufacturing, varios informes; Momento Económico no.49 (enero-febrero de 1990) UNAM NOTA: n.d.= No disponible. 
Sin embargo, la evidencia empírica está demostrando que una de las formas en que se logra elevar la productividad del trabajo es precisamente por medio de la segmentación del proceso productivo, la introducción de avances tecnológicos, y el mantenimiento de bajos salarios en las ZPE.

Particularmente a través de la informática, que ha revolucionado las normas de trabajo, se han refinado los métodos de la administración científica tanto en los procesos de trabajo con base tecnológica o intensivos en uso de fuerza de trabajo, sin necesidad de pagar salarios más altos.

A través de la especialización productiva, las mujeres en particular, han encontrado un potencial mercado de trabajo, de tal forma que en países como México éste tiende a incrementarse significativamente y a elevar el peso sobre la Población Económicamente Activa (PEA), particularmente en las principales ciudades de la frontera norte.

Esthela Gutiérrez (1985) señala que los cambios que se han impuesto sobre el consumo de la fuerza de trabajo se van a expresar en: 1) la magnitud de la fuerza de trabajo, 2) la sumisión del trabajo al capital, 3) la intensidad del desgaste físico y mental y 4) los requerimientos de la calificación del trabajo.

El avance tecnológico ha permitido la flexibilización del trabajo, que es lo que determina la magnitud de la fuerza de trabajo, además de transformar "el modo de trabajar que tiene profundas consecuencias sobre la lucha obrera, tanto dentro como fuera de la fábrica" (Laurell, 1983). Laurell sostiene que a pesar de que las razones que suscitan la prolongación de la jornada de trabajo sean de orden técnico, tienen repercusiones en el desgaste obrero, no sólo porque se trabaja durante más horas sino también debido a que la exposición al riesgo de trabajo se incrementa.

Bajo la nueva forma de organización del trabajo sufren modificaciones todos los mecanismos que antes regían el desempeño de actividades intensivas en mano de obra. La incorporación de nuevas tecnologías, además de estimular la adopción de actividades de manufactura, hace más complejas las tareas del ensamble, requiriendo con ello mayor capacitación y calificación de los obreros.

En cuanto al grado de calificación requerido, "la heterogeneidad tecnológica no es resultado del atraso-desarrollo, sino que obedece a las estrategias concretas de extracción de plusvalía que despliega en un momento histórico concreto el capital" (Laurell, 1983) y en este momento parte de esas estrategias es modificar el concepto de la capacitación-calificación.

La amplia difusión de los sistemas flexibles de organización del trabajo, ha propiciado un incremento sustancial en los niveles de productividad, revolucionando a la vez el contenido del trabajo, confiriéndole al trabajador, especialmente a las mujeres, un nuevo rol, en el que se le adjudica un mayor grado de responsabilidad y participación en la línea de producción. 
Lo anterior se logra particularmente a través de la adopción y generalización del sistema de "gestión de calidad total del proceso", que se aplica con relativa facilidad en pequeñas, medianas y grandes empresas.

Para que los obreros de línea logren una participación directa y sustantiva en el control de la calidad, es necesario que tengan un mayor grado de información respecto a la naturaleza del producto, fases del proceso, usos y aplicaciones y que estén preparados para actuar ante contingencias y cambios en la línea.

En un estudio de caso que incluyó a siete plantas maquiladoras en 1990 (Barajas, 1990), se percibió que el concepto de gestión de calidad total del proceso, había sido adoptado en todos los casos como una línea estratégica para buscar la máxima eficiencia; lo que varía en cada planta son las técnicas a las que se recurre para lograr dicho objetivo. Su adopción tiene su origen a partir de mediados de la década de los ochenta.

La incorporación de este nuevo esquema representa, en todos los casos estudiados, formas no tradicionales de organización de la producción y del trabajo, ya que desde el primer momento en que el obrero(a) se incorpora a la línea de producción, debe poner en juego toda su experiencia y habilidad, puesto que se le asignan las mismas responsabilidades que al resto de los operarios.

Particularmente, es en estos casos donde la destreza de las mujeres es estimulada por la puesta en marcha de diversas técnicas organizacionales como son el control estadístico de proceso, los círculos de calidad y equipos de trabajo, producción justo a tiempo y certificación de operaciones (ver cuadro 6).

\section{CONCLUSIONES}

Como se señaló al inicio de este artículo, el mayor énfasis del trabajo ha sido el resaltar los aspectos teóricos que dan sustento a la relación que se guarda entre reestructuración productiva, subcontratación internacional, cambio tecnológico y flexibilización del trabajo.

El caso particular de la industria maquiladora de exportación en México, ha servido como marco para intentar presentar esta relación, bajo la pretensión de que la evolución y desempeño de esta industria no sea vista en forma aislada y producto sólo de condiciones internas del país, sino fundamentalmente como resultado de una situación específica de la economía internacional.

La reestructuración productiva está involucrando una mayor segmentación de los procesos productivos, y ello a la vez estimula el crecimiento de las actividades de subcontratación en los países en desarrollo como México. Sin embargo, el propio concepto de la subcontratación también 
CUADRO 6. Sistemas y técnicas de gestión empresarial en las empresas visitadas

\begin{tabular}{|c|c|c|c|c|c|c|c|}
\hline Conceptos & $\begin{array}{l}\text { Levi } \\
\text { Mex }\end{array}$ & $\begin{array}{l}\text { Comair } \\
\text { Rotron }\end{array}$ & Plamex & $\begin{array}{l}\text { Video } \\
\text { Tec }\end{array}$ & Mexhon & $\begin{array}{l}\text { Compon. } \\
\text { La Mesa }\end{array}$ & Mabamex \\
\hline $\begin{array}{l}\text { Control Estadíst. } \\
\text { de Proceso }\end{array}$ & & $\mathrm{x}$ & $\mathrm{x}$ & $\mathrm{x}$ & $\mathrm{x}$ & $\mathbf{x}$ & \\
\hline $\begin{array}{l}\text { Círculos de calidad } \\
\text { y equipos de trabajo }\end{array}$ & $\mathrm{X}$ & & $\mathrm{x}$ & $\mathbf{X}$ & & $\mathbf{X}$ & $\mathrm{X}$ \\
\hline $\begin{array}{l}\text { Depto. de Ing. de } \\
\text { Diseño del Prod. } \\
\text { (variaciones y adapta- } \\
\text { ciones al producto) }\end{array}$ & $\mathrm{x}$ & $\mathrm{x}$ & & $\mathrm{x}$ & $\mathbf{x}$ & $\mathrm{X}$ & \\
\hline $\begin{array}{l}\text { Producción justo } \\
\text { a tiempo }\end{array}$ & $\mathrm{X}$ & & & $\mathbf{X}$ & $\mathbf{X}$ & $\mathbf{x}$ & $\mathrm{X}$ \\
\hline $\begin{array}{l}\text { Certificación de } \\
\text { operaciones }\end{array}$ & & & $\mathrm{x}$ & $\mathrm{x}$ & $\mathrm{x}$ & $\mathbf{x}$ & $\mathbf{X}$ \\
\hline $\begin{array}{l}\text { Depto. de Ing. Indus- } \\
\text { trial y Desarrollo }\end{array}$ & $x$ & $\mathbf{X}$ & $\mathbf{X}$ & & & $\mathbf{x}$ & \\
\hline
\end{tabular}

FUENTE: Elaboración propia a partir de la información obtenida en entrevistas a gerentes de las plantas seleccionadas, junio de 1990.

se modifica, toda vez que ya no se trata solamente de la relocalización de segmentos del proceso que enfatizan el uso de mano de obra, actualmente se incorporan nuevas tecnologías de base microelectrónica a la vez que se instrumentan los últimos sistemas organizacionales que han venido a revolucionar la manufactura.

En coincidencia con otros estudios, la "dualidad tecnológica" es característica en la industria maquiladora de exportación; es decir, la introducción de tecnología microelectrónica en algunas fases del proceso se combina con el ensamble simple de la maquiladora tradicional.

En la industria maquiladora también se han difundido rápidamente los nuevos sistemas organizacionales, incorporándose igual en pequeñas, medianas y grandes empresas; ha sido mucho más rápida la flexibilización del trabajo que la difusión de maquinaria y equipo microelectrónico.

Por último, cabe señalar que si bien es cierto que estas transformaciones se han dado en la industria maquiladora, los incrementos en productividad no han llegado acompañados de aumentos significativos en los salarios de los operadores directos, lo cual puede crear una distorsión en los términos del crecimiento de la maquiladora. 
En estos momentos de revisión de los distintos mecanismos que regirán a la inversión extranjera y la subcontratación entre otros, resulta importante definir mecanismos de retribución, reconocimiento y valorización de la fuerza de trabajo, que respondan a logros e incrementos en la productividad del trabajo de este tipo de inversión extranjera en el país.

\section{BIBLIOGRAFÍA}

BARAJAS, Rocío y Carmen Rodríguez. 1989. Mujer y trabajo en la industria maquiladora de exportación. (Documentos de trabajo), Fundación Friedrich Ebert, México.

- $\quad$ 1990. "La mujer ante la reconversión productiva: el caso de la maquiladora electrónica en Tijuana”. En: Bernardo González-Aréchiga y José Carlos Ramírez (Comp.). Subcontratación y empresas transnacionales. Apertura y reestructuración en la maquiladora. COLEF y Fundación Friedrich Ebert. México.

BOYER, Robert. 1990. "La información de la producción y la polivalencia", en: Esthela Gutiérrez (Coord.), La ocupación del futuro, flexibilización del trabajo y desreglamentación laboral. Fundación Ebert, Editorial Nueva Sociedad. México.

CASTELLS, Manuel y Laura D' Andrea Tyson. 1990. "El impacto creciente de la revolución tecnológica". Perspectiva Economica, (revista trimestral de la economía mundial) no. 70, 1990/I.

CORIAT, Benjamín. 1982. El taller y el cronómetro. Ensayo sobre el taylorismo y el fordismo y la producción en masa. Siglo XXI. Editor, España.

- 1988. "Automatización programable y productos diferenciados. Una visión sectorial de la automatización flexible y sus efectos". El movimiento obrero ante la reconversión productiva. Friedrich Ebert-CTMKES-OIT. México.

DRUKER F., Peter. 1990. "Las paradojas del desarrollo económico", Perspectivas económicas (revista trimestral de la economía mundial), no. 70.

GUTIÉRREZ Garza, Esthela. 1985. "La regulación competitiva como trasfondo del movimiento obrero". En: Reestructuración productiva y clase obrera. Testimonios de la crisis I. Siglo XXI editores. México.

LAURELL, Cristina. 1983. El desgaste obrero en México. Proceso de producción y salud, ERA, México. 
MERTENS, Leonard, 1988. "El movimiento obrero y las necesidades de capacitación ante la reconversión productiva", El movimiento obrero ante la reconversión productiva. Friedrich Ebert-CTMLES-OIT.

PIORE, Michael. 1990. "Dos concepciones sobre la flexibilidad del trabajo" en: Esthela Gutiérrez (Coord.). La ocupación del futuro. Flexibilización del trabajo y desreglamentación laboral. Fundación Ebert, Editorial Nueva Sociedad. 